Vol: 1(1), 2022

REST Publisher; ISBN: 978-81-948459-0-4

Website: http://restpublisher.com/book-series/materials-and-its-characterization/

\title{
Advanced Acoustic Emission Techniques for Failure Analysis In Concrete
}

\author{
*D. Monika, M. Mareeswaran \\ Department of Mechanical Engineering, M.E (Industrial Safety Engineering), Sri SaiRam Institute of Technology, \\ Chennai, Tamil Nadu, India. \\ *Corresponding author Email: sitp20is07@sairamit.edu.in
}

\begin{abstract}
To research fracture mechanisms in concrete, quantitative acoustic emission (AE) strategies may be implemented. In this context, a 3-D localization of the recorded AE events is required. In a first step, experiments have been carried out to assess the impact of various factors on the accuracy of localization consequences. AE sources had been either artificially generated on the surface of the specimens or received from loading assessments. An eightchannel acquisition unit turned into used to record AE parameters and waveforms. The first-class of arrival time determination was changed into found to be vital for the localization and may be improved with the employment of electricity primarily based set of rules. In a $2 d$ step selected AE activities, recorded in the course of pull-out exams, were analyzed quantitatively. With the help of a relative moment tensor inversion approach, AE source characteristics will be recovered.
\end{abstract}

\section{Introduction}

$\mathrm{AE}$ is defined as the spontaneous release of localized strain energy in a confused fabric ensuing, for example, from micro cracking and may be recorded by means of transducers (sensors) at the floor. AE evaluation is a beneficial method for the investigation of nearby harm in substances. one of the advantages compared to different NDE techniques are the possibility to observe harm approaches at some stage in the whole load history without any disturbance to the specimen. More state-ofthe-art evaluation procedures, such as fault-plane solution or moment tensor inversion strategies, are carried out to research the failure mechanisms of $\mathrm{AE}$ sources. Therefore, $\mathrm{AE}$ approach must be used in a quantitative way primarily based on the recorded floor displacement transients (waveforms). The use of six or more transducers is required to determine the radiation sample of a fashionable AE source. Fracture kind, length and orientation can be deduced. the premise for those quantitative techniques is a localization technique to extract the source coordinates of the AE events as it should be as viable. The objective of the assignment may be to design an SMS digital fire safety robot toolkit which can update the conventional hearth safety robot. The toolkit sends the hearth and sends SMS to the proprietor of the house, The gadget is made green by way of SIMs in order that the SMS can be acquired by using the number of devices forums in a locality using strategies of time department a couple of get admission to. Acoustic emissions are acoustic waves generated by using a rapid exchange inside the strain nation of a location in a material. Acoustic waves are certainly one of the non-electromagnetic techniques of transmitting energy through a material; the opposite approach is thermal diffusion. A distinction among the 2 is that thermal diffusion includes direct electricity transfer among man or woman atoms, while an acoustic wave transfers strength by way of a cooperative movement of many atoms. An acoustic wave can be as simple as a pressure pulse in fuel or liquid, or as complex as the various modes of transmission in a bounded solid. The technology of an acoustic wave invariably includes a massive vicinity of atoms. The maximum common acoustic waves are sound waves in air and water. these are produced by using the vibration of a surface consisting of the vocal apparatus of an animal, a leaf inside the wind, or the diaphragm of a speaker.

- Acoustic emission alerts generate complexity because the wave travels via the medium. Underneath standing acoustic emission alerts calls for expertise of the wave traits, starting with the homes of the medium thru which the wave travels.

- All substances are collections of atoms held together through appealing forces even as concurrently prevented, through short-variety repulsive forces, from approaching each different too carefully.

- The superposition of those force's consequences in an equilibrium role for the atom at its lowest strength, the fabric's maximum strong configuration.

- In crystals, for instance, long-variety forces among the atoms bring about defined locations for those equilibrium positions. The result is a crystal structure. In a liquid the shape is amorphous, but an approximate distance among atoms effects in a uniform density.

- The acoustic homes of a material depend upon its density and the lengthy variety coupling constants among its atoms. The lengthy-range correlations in an acoustic wave result in many atoms in a small area being displaced in the same route from their equilibrium positions.

- This displacement is a local dynamic pressure in the crystal; the stress's course and importance are constantly converting because the atoms circulate. while the atomic motion is pseudo-oscillatory, so is the pressure.

- An acoustic wave is an oscillating stress transferring via a cloth. due to the fact stress and strain are usually 
without delay related to cloth, there is additionally an oscillating pressure subject. therefore, an acoustic wave may be described as both dynamic strain and pressure field in a material.

\section{Methodology}

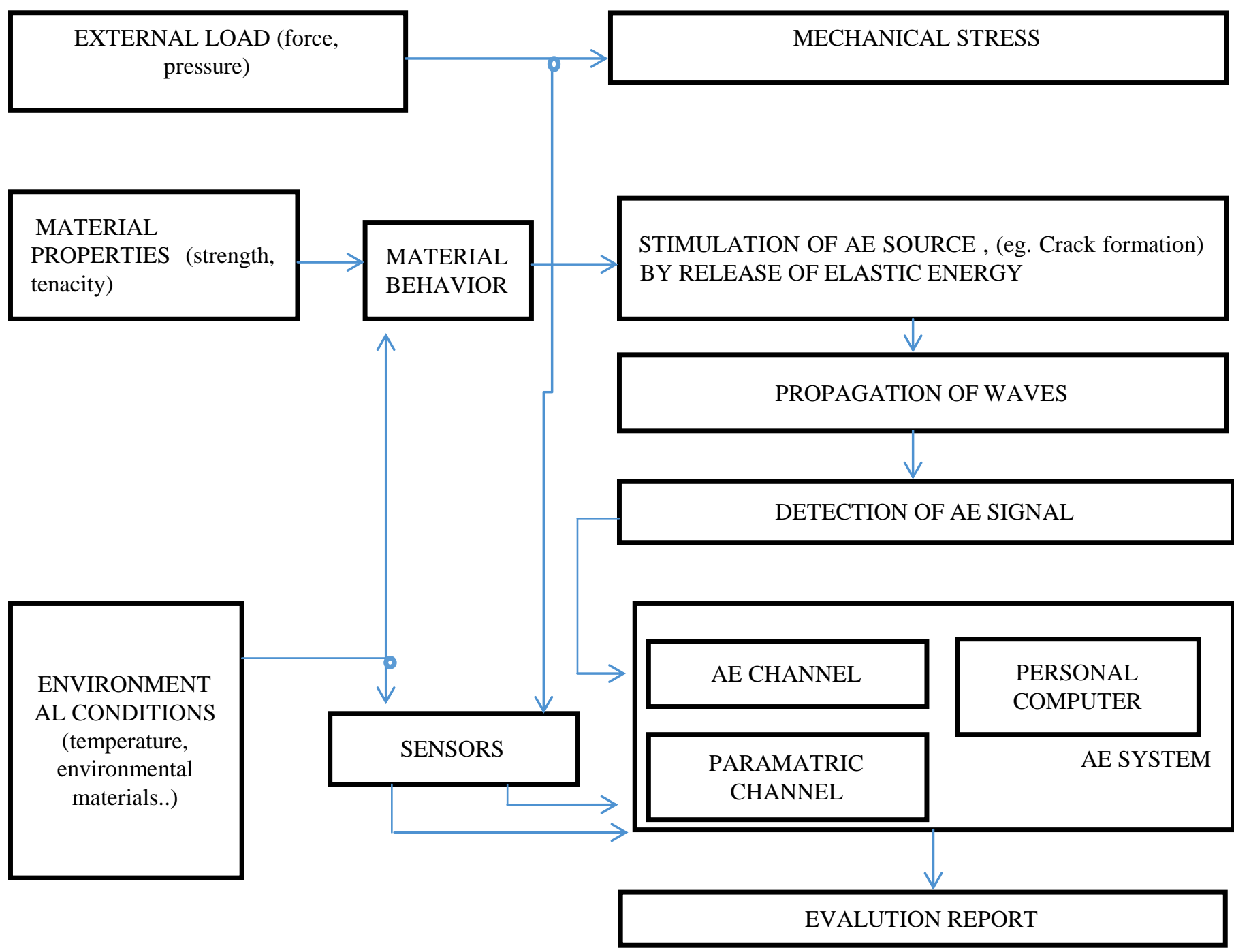

3. Material Requirements

1. Portland cement aggregate $-330 \mathrm{Kg}$

2. Piezoelectric transducers -8

3. Personal Computer

1. Pull-out Test

\section{Tests Conducted}

2. Proof Test

3. Failure Test

4. Fatigue Test

\section{Experimental procedure}

1. Affects at the accuracy and reliability of localization consequences in concrete have been to be investigated. It changed into, consequently, important to recognize the real supply locations.

2. Experiments were done with artificial assets (pencil breaks) at described positions at the surface of the specimen.

3. Loading checks had been designed to provide AE because of cracking or friction in small and predictableareas.

4. For all specimen's ordinary density concrete with $330 \mathrm{~kg}$ of Portland cement in line with m three and water cement ratio of 0.47 was used. the mixture share turned into 0.35:0.3:0.35 for aggregate sizes 0 -4:4-8: eight-16 $\mathrm{mm}$.

5. An industrial AE measuring gadget turned into used to accumulate AE facts throughout the assessments. eight channels with transducers, preamplifiers and A/D converters were employed concurrently. 
6. Piezoelectric transducers (PZT) with a extensive-banded and calibrated frequency reaction among $50 \mathrm{and} 250 \mathrm{kHz}$ and a diameter of $13 \mathrm{~mm}$ had been applied. besides traditional AE parameters additionally brief waveforms were saved over a duration of $205 \mathrm{mms}$ with a digitizing fee of $10 \mathrm{MHz}$

7. Source places of $\mathrm{AE}$ activities recorded through all 8 transducers have been calculated online through the acquisition unit and in a next evaluation the use of HypoAE. For the p-wave pace a fee of $4.8 \pm 0.1 \mathrm{~m} / \mathrm{ms}$ turned into decided in initial tests and discovered to be equal inside the given bandwidth for all specimens.

8. In a first experiment, localization outcomes of pencil spoil on a concrete cube (side length $200 \mathrm{~mm}$ )had been as compared with the actual positions.

9. The supply places displayed have been calculated online the use of the first threshold crossings as the arrival times.

10. For the locations was implemented to decide the arrival times from the recorded waveforms best a small improvement became performed and localization mistakes ranged for both algorithms from 0 to $5 \mathrm{~mm}$.

11. The respective distributions are plotted, where the vertical axis is normalized to the absolute variety of localization results.

12. A sequence of pullout tests was performed to study AE alerts resulting from the interplay of metallic and concrete in the bond zone. 4 concrete cubes with aspect lengths of $200 \mathrm{~mm}$ and a focused re-bar f $14 \mathrm{~mm}$ were used (discern three). The bond period turned into chosen as $42 \mathrm{~mm}$ which equals three rebar-diameters or 5 ribspacings.

13. AE indicators had been first because of the lack of adherence on the steel-concrete interface and then via shearing action in the concrete among the steel ribs. The real failure sector became constrained to the very near place of the bond surface.

\section{Conclusion}

AE experiments with reinforced concrete had been performed a good way to examine unique affects at the accuracy of localization effects. The nice of arrival time dedication become determined to be critical and could be stepped forward with the employment of a power-based totally algorithm, carried out in the WinPecker application. The relative second tensor inversion turned into carried out to research $\mathrm{AE}$ activities recorded for the duration of pull-out assessments. The primary instructions of the calculated moment tensor as well as its decomposition into isostatic and deviatoric additives allowed one to signify exclusive supply mechanisms. only some consequences have been obtained so far but the agreement with anticipated failure mechanisms is promising. issues had been encountered with the time-eating and not usually the unambiguous dedication of the p-wave amplitudes used for the inversion

\section{References}

[1]. Alan G.bettiee.;acoustic emission for non-destructive testing of structure using source locationTechnique , sandia report ,SAND2013-7779

[2]. Aki, K.; Richards, P.: Quantitative seismology - theory and methods. Allan Cox; W. H. Freemanand Company, San Francisco (1980), 932 pp.

[3]. Dahm, T.: Relative moment tensor inversion based on ray theory: theory and synthetictests. Geophys. J. Int. 124 (1996), pp. 245-257.

[4]. Grosse, C.: Grundlagen der Inversion des Moment tensors zur Analyse von Schallemissions-quellen. In: "Werkstoffe und Werkstoffprüfung", Festschrift zum 60. Geb. von Prof. Reinhardt(Hrsg. C. Grosse), Libri BOD (1999), pp. 82-105.

[5]. Grosse, C.; Weiler, B.; Reinhardt, H.: Relative moment tensor inversion applied to concretefracture tests. J. of Ac. Emission 14 (1996), No. 3-4, pp. 74-87.

[6]. Grosse, C.; Reinhardt, H.; Dahm, T.: Localization and classification of fracture types in concretewith quantitative acoustic emission measurement techniques. NDT\&E International, Vol. 30 (1997),No. 4, pp. 223-230.

[7]. Grosse, C.; Reinhardt, H.: Entwicklung eines Algorithmus zur automatischen Lokalisierung vonSchallemissionsquellen. Die Materialprüfung 41 (1999), pp. 342-347.

[8]. Grosse, C.: WinPecker - Programm zur vollautomatischen dreidimensionalen Lokalisierung vonSchallemissionsquellen. 12. Kolloquium Schallemission, DGZfP Berichtsband BB 72 (2000), pp.191-204.

[9]. Köppel, S.; Vogel, T.: Localization and identification of cracking mechanisms in reinforced concrete using acoustic emission analysis. Proc. 4. Int. Conf. on Bridge Management, Surrey (2000),pp 88-95.

[10]. Landis, E.; Ouyang, C.; Shah, S.P.: Automated determination of first p-wave arrival and acousticemission source location. J. of Ac. Emission 10 (1991), No. 1-2, pp. 97-103.

[11]. Landis, E.; Shah S.P.; Signal analysis for quantitative acoustic emission testing. NondestructiveTesting Methods; ASCE; New York (1995), pp. 45-56.

[12]. Ohtsu, M.; Shigeishi, M.; Iwase, H.; Koyanagi, W.: Determination of crack location, type andorientation in concrete structures by acoustic emission. Mag. of Concrete Research 43 (1991), No.155, pp. 127-134.

[13]. Ohtsu, M.; Okamoto, T.; Yuyama, S.: Moment tensor analysis of acoustic emission for crackingmechanisms in concrete. ACI Structural Journal 95 (1998), No. 2, pp. 87-95.

[14]. Oncescu, L.; C. Grosse:HYPO AE - A program for the localization of hypocenters of acousticemissions. Software für PC and Workstation, Rev. 2.0 (1996), 15 pp. 